\title{
Participación ciudadana y movilidad sostenible: el caso del área metropolitana de Concepción, Chile
}

\author{
Citizen participation and sustainable mobility: the case of the metropolitan area of Concepción, Chile
}

\section{Javier León Aravena*, Francisco Núñez Cerda* y Elías Albornoz del Valle*}

Recibido: 22 de enero de 2019

Aceptado: 27 de mayo de 2019

\section{Resumen}

La movilidad es un problema multifactorial. Entre sus aspectos figura la subjetividad de los ciudadanos, asociada a percepciones de los problemas que detectan, sensaciones y emociones que les provoca la experiencia de movilidad. Sin embargo, estos elementos, no son apropiadamente considerados en la formulación de los respectivos planes. La pregunta de investigación que se plantea es, si la participación además de identificar elementos objetivos para un plan, también lo puede hacer con los elementos subjetivos. El artículo describe cómo se exploraron e identificaron dichos aspectos para ser incluidos en la Formulación del Plan de Movilidad Sostenible para el Área Metropolitana de Concepción, Chile, realizado el año 2017. Se utilizó una metodología participativa implementada a través de talleres y encuestas aplicadas a informantes claves. Los datos fueron analizados de acuerdo triangulación metodológica y criterios de movilidad sostenible. Uno de los hallazgos destacados, es la funcionalidad y eficacia que la metodología participativa logra para identificar el papel de las emociones en la planificación. Otro hallazgo es la mención en un $70 \%$ de emociones negativas. Tal resultado, plantea la preocupación con relación a como se están llevando a cabo las actuales políticas y procedimientos de diseño e implementación de planes de movilidad, los que no consideran adecuadamente estos aspectos.

Palabras clave: área Metropolitana, movilidad sostenible, participación ciudadana.

\begin{abstract}
Urban growth places mobility as a multifactor problem and complex to solve. Among its less weighted aspects is the subjectivity of the phenomenon, specifically how people perceive their mobility and how participatory they can affect the definition of the city and transport planning. In this problematizing context emerges the study "Formulation of a Sustainable Mobility Plan for the Metropolitan Area of Concepción", applied in the Biobío Region, Chile, and based on a citizen participation approach. Among its most outstanding findings is the role of the emotions of individuals, specifically what refers to the fear and insecurity that condition their movement through the city, beyond the situation of transport and infrastructure that it has. The foregoing would invite to rethink the meaning of the current plans and policies of urban planning, in order to specify a sustainable model of mobility.
\end{abstract}

Keywords: citizen participation, metropolitan Area, sustainable mobility.

* Filiación: Universidad del Bío-Bío, Concepción, Chile. Contacto: jleon@ubiobio.cl , fnunez@ubiobio.cl, ealborno@ubiobio.cl

Cómo citar: León Aravena, J., Núñez Cerda, F. y Albornoz del Valle, E. (2019). Participación ciudadana y movilidad sostenible: el caso del área metropolitana de Concepción, Chile. Revista de Urbanismo, 40, 1-18. https://doi.org/10.5354/0717$\underline{5051.2018 .52227}$ 


\section{Introducción}

La movilidad urbana comienza a ser concebida como la suma de los desplazamientos individuales, a través de lo cual se posibilita el acceso al mercado de trabajo, bienes y servicios (Gasparini \& Guidicini, 1990), no definiéndose únicamente por la totalidad de los desplazamientos, debido a que la movilidad urbana constituye un modo de funcionamiento de la sociedad (Bourdin, 1999). De esta forma, la definición de movilidad urbana adquiere más elementos y se definiría como la suma de desplazamientos de personas, bienes y de informaciones, respondiendo a la necesidad de desplazarse en un espacio determinado, incluyendo todos los efectos positivos y negativos que estos desplazamientos producen en la ciudad (Escudero, 2004). Actualmente se presenta como uno de los problemas más importantes de la vida social (Osorio y García, 2017), constituyéndose como un fenómeno multidimensional y factor en sí mismo de desarrollo político, económico y sociocultural (Lange, 2011). Su origen, como fenómeno, se fundamenta en la articulación entre el crecimiento y consolidación de las ciudades modernas, quienes progresivamente debieron enfrentar problemas como el desborde demográfico, la saturación de los servicios, la degradación ambiental y la inseguridad ciudadana, factores a los que se sumó una lenta respuesta del Estado frente a dichos dilemas (Dangond, Jolly, Monteoliva y Rojas, 2011).

Esa génesis sitúa como principio rector del fenómeno la relación entre movilidad y la acción estatal, desmarcando esta problemática de una mera concepción urbanística referida al transporte público, entendiéndola, en cambio, como un objeto inserto en el quehacer político-administrativo. En este sentido, podría afirmarse que el objetivo de la movilidad no se enfoca específicamente en cuantificar y predecir viajes, sino más bien en el cómo los habitantes de una urbe o sector acceden a los diversos recursos, tales como servicios públicos, esparcimiento, trabajo o conocimiento, entre otros (Mendoza, 2013).

Atendiendo a las externalidades negativas producidas por el acto masivo de desplazarse, en un comienzo ambientales, y sin perder de vista las sociales y económicas (Sanz, 1997), se incluye en su conceptualización el paradigma de la sostenibilidad. La movilidad sostenible se definiría como la capacidad para satisfacer las necesidades de la sociedad para moverse libremente, acceder, comunicarse, comercializar y establecer relaciones, sin sacrificar otros valores humanos o ecológicos básicos actuales o del futuro (WBCSD, 2001). La definición anterior, claramente destaca aspectos sociales, diferenciándola de aspectos puramente técnicos y preocupaciones dirigidas solamente a la implementación de sistemas de transporte sostenibles, que hagan posible en el tiempo, la satisfacción de necesidades. De esta forma nacerían los planes de movilidad sostenible (PMUS) a nivel europeo, donde las personas son el foco principal de éstos; ya se trate de viajeros, empresarios, consumidores, clientes, vecinos o cualquier otro carácter que defina a los sujetos. La preparación de un PMUS significa así: Planificación para la gente, centrándose en el funcionamiento de la ciudad (Unión Europea, 2017).

El derecho a una movilidad sostenible, considerando los aspectos sociales, ambientales y económicos, es un desafío para las sociedades modernas, más aún considerando las nuevas necesidades que acompañan el anhelado desarrollo económico. En Chile, a pesar de los esfuerzos por abordar de mejor forma los problemas de movilidad urbana, el acceso a actividades y servicios se torna cada vez más difícil debido a los acelerados cambios de escala que actualmente experimentan las ciudades metropolitanas, aún carentes de una institucionalidad que regule los mecanismos de movilidad (Silva, 2017). Lo anterior, marca una notoria diferencia con otros países más adelantados en la materia, en que los planes de movilidad sostenible son requisitos legales, constituyendo su formulación un desafío conceptual y práctico (Mello, 2017).

La revisión de la literatura a nivel latinoamericano revela una interesante producción científica al respecto, destacando en este sentido los casos de Colombia y Brasil, países en los que la movilidad se ha perfilado como un referente de reflexión académica y política (Alcántara, 2010; Cunha, 2000; Dangond et al., 2011; Rolnik, 2009). EI panorama en Chile, en cambio, presenta una producción escasa y mayormente concentrada en la capital, Santiago, omitiéndose así la diversidad, riqueza y necesidades presentes en otras regiones del país, aun cuando existen por lo menos dos grandes áreas metropolitanas adicionales, como son Valparaíso y Concepción. No obstante, es necesario reconocer la preocupación 
generada a nivel político, destacando en ese sentido documentos como el elaborado en 2014 por la Comisión Asesora Presidencial y titulado Problemas de la Movilidad Urbana: Estrategia y Medidas para su Mitigación, y cuyo objetivo fue abordar los temas de transporte con relación a la calidad de vida de la población, la productividad y la competitividad económica. En la misma línea se redactó en 2016 Chile, Informe Nacional Habitat III, en el que se declaró explícitamente la intención del Estado de avanzar en temas de movilidad no sólo en Santiago, sino también en las dos áreas metropolitanas recién mencionadas.

Sin embargo, ni en el ámbito político ni científico se ha tocado con mayor profundidad la cuestión referida a la movilidad y la participación ciudadana en el país. De hecho, la producción posible de encontrar a nivel local, y más allá de la rigurosidad y profesionalismo con que ha sido llevada a cabo, no aborda de manera particular este problema y su relación sostenible con la ciudadanía. Tal es el caso, por ejemplo, del estudio de Francisco Maturana, Andrés Rojas y Roberto Salas (2017), llevado a cabo en la ciudad de Temuco y en el que se toca la movilidad más bien desde una perspectiva espacial y administrativa.

Como una excepción a este vacío se constituye el diagnóstico participativo realizado en 2017 en once comunas del área Metropolitana de Concepción, región del Biobío, en el marco del Estudio Formulación de un Plan de Movilidad Sostenible para el Área Metropolitana de Concepción. La formulación fue encargada por el Gobierno Regional del Biobío a la Universidad del Bío-Bío y cuyo objetivo fue identificar los problemas, preferencias, emociones y necesidades de desplazamiento que experimentan los habitantes de comunas adscritas a esta área Metropolitana, a efecto de detectar los niveles de sostenibilidad social del actual sistema de movilidad metropolitano y sobre esa base formular un plan sostenible.

Para ello se llevó a cabo un trabajo enfocado en una perspectiva ciudadana de la movilidad, rescatando visiones, conocimientos y expectativas del hábitat de quienes se desplazan (Herrmann, 2016). En este trabajo se dio protagonismo a elementos subjetivos, normalmente desechados de la planificación tradicional, tales como: las necesidades sentidas, emociones o deseos de las personas. Ello como una forma de dotar el fenómeno no solo de una mayor sustentabilidad, sino también de humanizar la mirada sobre la movilidad (Gutiérrez, 2012).

La pregunta de investigación que se plantea en este contexto es si un proceso participativo además de recoger apropiadamente elementos objetivos asociados a la movilidad de los ciudadanos, también lo puede hacer en relación a elementos subjetivos asociados, tanto de tipo individual y colectivo, a efecto de ser aplicados para la formulación de un plan de movilidad sostenible.

El presente artículo tiene como objetivo exponer la experiencia de este diagnóstico con el fin de escudriñar en la importancia y utilidad del componente personal y subjetivo en la búsqueda de un modelo de movilidad sostenible. Ello junto con destacar el valor que amerita el desarrollo de este tipo de experiencias en un contexto descentralizado, el cual permita obtener una visión más amplia del fenómeno en cuestión basado en la pertinencia territorial de la mirada del estudio.

\section{Aproximaciones Conceptuales}

\section{Personas y Movilidad}

La movilidad como fenómeno social adquiere impulso gracias a las investigaciones de Jaques Lévy (2000). Este autor incorporó tres dimensiones fundamentales para su comprensión: La primera está relacionada con oferta de acceso, entendida como una potencialidad; la segunda apunta a las competencias del individuo para efectuar la movilidad y acceder a las actividades; la tercera, en tanto, plantea movilidad como capital, constituyéndose como el conjunto de recursos necesarios para realizar el desplazamiento. La conjugación de estas tres dimensiones determinaría la elección de la mejor estrategia por parte del individuo para desplazarse al interior de la sociedad.

Al respecto, es importante que la movilidad no sea restringida a temas de infraestructura vial y/o estudios propios del transporte. Hacerlo conlleva a eliminar de su análisis el necesario rol de los sujetos y los grupos sociales en la producción y transformación del espacio urbano (Ramírez, 2007) lo que, a su vez, conduce a la implementación de soluciones de corto plazo y otros fenómenos que complejizan aún más el problema, tales como el crecimiento urbano descontrolado o el incremento en el uso de automóvil. Actualmente esta perspectiva cortoplacista física-causal goza de aceptación 
para decisiones políticas y de planificación urbana, por su eficacia y métodos para su validación científica (Cerda y Marmolejo, 2010). Lo que se espera, en cambio, es situar la movilidad en un contexto social y sistémico de planificación, el cual aborde problemas sociales, económicos, políticos y físicos vinculados a la circulación de las personas (ONU-Habitat, 2015).

De acuerdo a Kaufman, Bergman y Joye (2004), esta visión social de la movilidad se conceptualiza como motilidad, siendo la capacidad de entidades para moverse en el espacio geográfico. Tal conceptualización, está dada por la identificación de factores, parámetros y variables para recorrer el espacio-tiempo, bajo una dinámica socioeconómica. A partir de esto, motilidad se explica en tres factores: a) Accesibilidad: disposición de un servicio; b) Capacidades sociales: estrategias alcanzables, según las características del individuo para utilizar la oferta disponible; y c) Apropiación: cómo las opciones disponibles son utilizadas para materializar el desplazamiento (Kaufmann \& Widmer, 2006).

Bajo esta perspectiva, existen dos elementos explicativos claves: las competencias de los individuos y como éstos experimentan la apropiación misma del espacio, (Cerda y Marmolejo, 2010). Esto último se relaciona con la percepción individual y como ellos interpretan su proceso decisional, según sus capacidades de acceso al lugar de destino. Las decisiones podrían estar condicionadas a una serie de alternativas disponibles para el individuo para su desplazamiento y, por tanto, atingentes a su capacidad de realización y acceso satisfecho. Este planteamiento, sin embargo, si bien destaca el rol de las decisiones o las preferencias, no define atributos relacionales y colectivos que pueden surgir de la participación ciudadana y procesos más amplios de gobernanza.

De este modo, uno de los temas pendientes más necesarios de resolver es la incorporación de las aspiraciones y derechos ciudadanos en un enfoque sustentable de movilidad (Herce, 2009; Dangond, et al., 2011; Vega, 2016). Con ello se busca integrar una serie de aspectos de índole social, cultural, económica y política surgidos de la participación (Quintero, 2017), premisa coherente con la instauración de una apropiada gobernanza urbana o metropolitana (ONU, 2016).

\section{Participación, ciudad y movilidad}

En un mundo donde casi la mitad de la población habita en espacios urbanos resulta lógico que el crecimiento de las ciudades se haya acompañado de una constante complejización de los fenómenos que allí ocurren. Al respecto, las áreas metropolitanas resultan paradigmáticas dadas sus continuas reestructuraciones territoriales, lo que conlleva alteraciones del entorno material, con consecuencias sobre el hábitat social. Esto ha generado problemáticas tales como: la segregación espacial, el empobrecimiento y deterioro de las relaciones sociales, entre otras. Dichas situaciones de carácter cotidiano repercuten en la calidad de vida de las personas que residen en estos lugares.

Ante la persistencia de estas situaciones, es posible concluir que la respuesta de las políticas públicas, y los criterios de la planificación urbana en particular, no han sido suficientes para resolverlas, cuestión que denota un abordaje ineficiente por parte del Estado. Frente a ello, ha sido la propia sociedad civil, quien, de manera espontánea o institucionalizada, se ha visto en la necesidad de involucrarse directamente en la búsqueda de soluciones para estos asuntos, producto de lo cual se podría afirmar que la extensión y complejidad de los fenómenos urbanoterritoriales ha derivado en la puesta en escena de un ejercicio legítimo de ciudadanía (Alessandri, 2014).

Desde una perspectiva liberal e histórica, es posible enlazar el concepto de ciudadanía con el de participación, entendida esta última no solo como una condición, sino también como una actividad capaz de vincular la práctica política, el desarrollo económico y la sociedad como Estado organizado (Giraldo-Zuluaga, 2015; Wiener, 1997). La participación implica la injerencia de los ciudadanos y la sociedad en asuntos que se busca resolver de manera conjunta, o hacer confluir en ellos, una serie de voluntades dispersas que es conveniente articular y materializar en las decisiones públicas (Fernández, 2014).

En un sentido clásico, la participación puede ser concebida como una forma a través de la cual los individuos intervienen en actividades del quehacer público representando los intereses de la sociedad de la que hacen parte (Cunill, 1991). La participación es un concepto sujeto a diversas manifestaciones e interpretaciones, pudiendo presentarse en formatos pasivos, restringidos, deliberativos, institucionales, etc. 
(Welp, 2016). La forma que ella adquiera estará determinada por la existencia (o no) de mecanismos y procedimientos que estipulen su práctica y alcance (Garrido-Vergara, Valderrama y Peñafiel, 2016). No obstante, para fines de este artículo, requerimos abordarla desde una acepción resolutiva y enmarcada dentro de la distinción entre lo estatal y público (De la Maza, 2011), básicamente porque ello apunta a vincular la acción ciudadana, con la acción del Estado (Baño, 1998). Es importante señalar que, aun cuando la participación no depende solo de las posibilidades que la institucionalidad provea, menos aun cuando su potencialidad se ha encontrado históricamente en disputa con el actor Estado (Sánchez y Gutiérrez, 2014), vale la pena preguntarse respecto de su configuración y el impacto que ella puede tener en las estructuras habilitadas evaluando su eficacia en la gestión integral de las políticas públicas.

Por ello, contextualizando el marco conceptual con el objeto de estudio de este artículo, si se entiende a la ciudad como una construcción social, elaborada por los sujetos en términos materiales (edificaciones, calles, etc.) e inmateriales (su conciencia) (Vergara, Rozas y Zunino, 2013), es posible dimensionar la importancia y el alcance que puede tener la participación cuando se encuentra asociada a procesos de planificación y decisión. Siendo así, la participación debe pensarse en función del sentido evolutivo que ha adquirido el derecho a la ciudad (Sagaris y London, 2017).

Desde esta perspectiva, la participación se constituye como una instancia de continuo aprendizaje, comunitario e institucional, y productora de múltiples beneficios (Poduje, 2008). Cuando ella es efectiva y debidamente articulada con el rol de otros actores, es posible generar un trabajo en red capaz de incrementar competencias y recursos, y, por ende, extender los alcances derivados de esta asociación sinérgica (Aguilar, 2014). Entre los principales beneficios de la participación asociados a la problemática urbana destacan el mejoramiento de las condiciones de vida colectiva, al orientar las intervenciones y regulaciones a través del diálogo y la negociación, como así mismo una mejora en la calidad de los proyectos al añadir nuevo valor al uso de los espacios, interacciones y relaciones que se gesten en su interior (Poduje, 2008; Sánchez y Gutiérrez, 2014).
Por supuesto, la participación tampoco se encuentra exenta de críticas. Entre ellas es posible aludir su carácter transitorio y fragmentado, sino es parte de una perspectiva integral de la gestión de las políticas públicas y metodológicamente bien diseñada como proceso. Por otro lado, no siempre existen garantías respecto a su real incidencia en las decisiones político-institucionales, más aún cuando el Estado históricamente se ha mostrado reacio a considerar a los ciudadanos como actores relevantes al momento de planificar las ciudades (Poduje, 2008; Sagaris y London, 2017). No obstante, lo cierto es que la incidencia en planes y programas de la participación, si bien es una condición necesaria, tampoco es suficiente por si sola para garantizar una participación democrática (Welp, 2016), más aun, en el contexto de sociedades caracterizadas por situaciones de segregación y exclusión (Jiménez, 2008). Dicho de otro modo, la participación en la gestión de las políticas no llegará a buen puerto, si persisten espacios de segregación y exclusión.

En el contexto latinoamericano, y también en gran parte del mundo, la participación ciudadana ha experimentado un incremento asociada a la gestión de las políticas, al tiempo que ha disminuido la afiliación en partidos políticos y ha aumentado la desconfianza en los gobiernos (Welp, 2016). Sin embargo, es importante señalar que las instancias institucionalizadas de participación no han surgido necesariamente a partir de reflexiones o debates políticos progresistas, sino más bien como resultado de la presión y lucha entablada por los distintos actores involucrados por mejorar sus condiciones de vida, destacando en este sentido la ciudadanía y los movimientos sociales (Schneider y Welp, 2015). En cuanto a la participación para temas de planificación urbana, existen experiencias interesantes, como el ejemplo de Brasil, con la inclusión del derecho social a la ciudad y los presupuestos participativos que buscan favorecer a grupos sociales vulnerables (Janoschka, 2011). No obstante, en general, se ha visto que la cantidad de mecanismos institucionales generados para la participación en los distintos países de la región no es directamente proporcional a su impacto ni con la posibilidad de una práctica realmente deliberativa (Garrido-Vergara et al., 2016).

En Chile los principales desafíos en materia de participación apuntan a su efectiva integración en la 
racionalidad y ciclo de las políticas públicas, cuestión que requiere fomentar y generar estructuras que la institucionalicen. Junto a ello, superar el proteccionismo de una política que basa su legitimidad democrática principalmente en mecanismos de representación, y que por tanto, cree poder prescindir de la ciudadanía, delimitando su participación a los comicios. Finalmente, salvaguardar las situaciones de exclusión que se pueden dar en el espacio mismo de participación, a propósito de los desbalances de poder, problemas que demanda establecer garantías mínimas de acceso democrático (De la Maza, 2008).

Por lo anteriormente mencionado, es que el estudio de la implementación de procesos participativos en la planificación es relevante, y en este caso en el marco de un fenómeno complejo, como la planificación de la movilidad sostenible. Para una planificación pública, con poca tradición en participación ciudadana en Chile, resulta un cambio de paradigma el incorporarla e indagar no solo aspectos formales y objetivos, sino que aquellos menos considerados en la gestión tradicionales, tales como las emociones, deseos y necesidades sentidas por las comunidades y las personas.

Esto por cuanto en las metrópolis y centros urbanos actuales, y dado su constante crecimiento, existen sectores de los territorios que quedan desconectados, sin servicios y con múltiples carencias, siendo muchas veces los propios ciudadanos los que instalan el tema de movilidad dentro de la agenda pública con miras a solucionar sus problemas (Espluga, Cebollada y MirallesGuash, 2008; Miralles-Guash, C., Cebollada, A. y Requena, R. 2010). Parece importante destacar que la movilidad sostenible incorpora la complejidad de la expectativas y acciones ciudadanas para el desarrollo de los planes de movilidad urbana. Las experiencias internacionales enseñan que la movilidad es una preocupación para los ciudadanos y que estos desarrollan estrategias para mejorarla, y para ello la participación en la planificación se evalúa como fundamental.

\section{El Área Metropolitana de Concepción (AMC)}

Un área metropolitana puede ser definida como una zona "urbana polinuclear que conforma un mercado unitario de residencia y trabajo, que refleja a la vez el aumento de escala del espacio de vida colectivo y las diferentes estrategias espaciales de los agentes económicos" (Feria, 2008 , p. 50). La escala de interacciones, intensidad y diversidad de fenómenos que se gestan al interior de estas conformaciones puede ser explicado por diversos factores, tales como el acelerado crecimiento de la población, los procesos de sub-urbanización, la desconcentración de las actividades económicas y las diferencias en los tipos de hogares, entre otros (Truffello e Hidalgo, 2015).

De acuerdo al Instituto Nacional de Estadísticas (2018), aproximadamente el $87,4 \%$ de la población de Chile vive en localidades urbanas, y aproximadamente el $63 \%$ de esas familias vive en las ocho aglomeraciones que son consideradas Áreas Metropolitanas. De éstas, la mayor es Santiago, seguida por Concepción y Valparaíso, respectivamente. Otro dato relevante entregado por Hidalgo y Arenas (2009) vinculado al desarrollo económico, y producto de éste, el país se encontraría viviendo un proceso de metropolización de los territorios urbanos.

El AMC, también conocida como Gran Concepción, comenzó a gestarse a fines de la década de 1950, producto de un proceso de industrialización que atrajo población del campo a la ciudad, lo que con el paso de los años conllevó a su expansión física y la intensificación de los procesos de interacción y conurbación ocurridos en su interior (Salinas y Pérez, 2011). Actualmente, y considerando todas las dificultades que ha implicado la definición de las localidades en cuestión. EI AMC se encuentra constituido por once comunas: Chiguayante, Concepción, Coronel, Hualpén, Hualqui, Lota, Penco, Santa Juana, San Pedro de la Paz, Talcahuano y Tomé (Figura 1). Según el censo de 2017 (INE, 2018) estas comunas suman 985.044 habitantes, aproximadamente el $50 \%$ de la población regional, distribuidos en una superficie de $2.830,4 \mathrm{Km}^{2}$, posicionándose como la segunda zona metropolitana en extensión del país. 


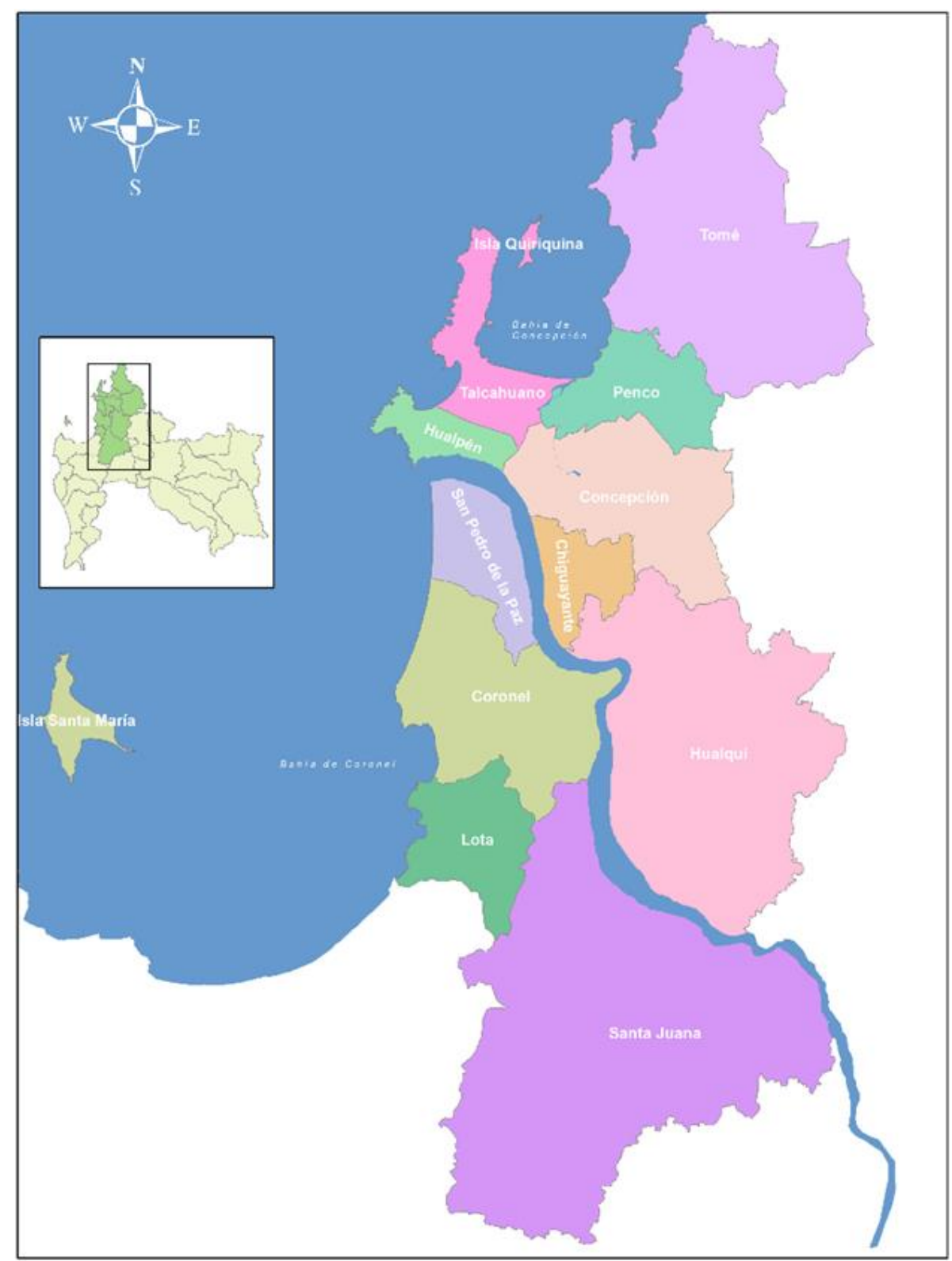

Figura 1. Área Metropolitana de Concepción.

Fuente: Universidad del Bío Bío (2018). Informe Propuesta de Plan de Movilidad Sostenible para el Área Metropolitana de Concepción.

Se trata de un área metropolitana de servicios complejos, proporcionados por la escala y especialización de sus actividades, fundamentada en el desarrollo industrial de gran envergadura, la educación superior, así como por su carácter de polo comercial y político administrativo del centro sur de Chile. La creciente expansión urbana y el constante proceso de conurbación han evidenciado el carácter unitario y metropolitano de esta zona, lo que ha evidenciado la necesidad de llevar a cabo una planificación integral que considere a todas las comunas que hacen parte de ella. Esto no es fácil si se considera su constante expansión, la cual actualmente abarca ciudades tan alejadas como Santa Juana, Lota y Tomé. No obstante, lo concreto es que el Plan Regulador Metropolitano de Concepción, vigente desde el año 2003, plantea la visión coordinada y el funcionamiento conjunto de este territorio.

La complejidad asociada al proceso mismo de metropolización ha decantado, al interior del AMC, en diversos fenómenos propios del desarrollo y el 
crecimiento. En este sentido, destacan, entre otros, problemas como el impacto medioambiental por la ocupación del territorio (Aliste, 2014) y la configuración de los asentamientos humanos, determinados en este caso por cuestiones como la densidad y la segregación socioespacial de sus habitantes, lo que a su vez ha dado origen a problemas como el uso de transporte y la huella ecológica vinculada a tales condiciones (Muñiz, et al., 2016).

A lo anterior se suman las condiciones específicas. Geográficamente, la ciudad se ha desarrollado de manera preferentemente longitudinal, ocupando por completo el área plana disponible; definida en su ancho por el cerro Caracol, los cerros isla Chepe y la Pólvora. Su desarrollo en extensión ha sido acotado por el río Bío-Bío que recorre las comunas de Santa Juana, Coronel, Hualqui, Concepción, Talcahuano, San Pedro de la Paz y Hualpén, y por las áreas cercanas al río Andalién. Por otro lado, el AMC también está acotada por las bahías de Concepción y San Vicente, bordes costeros de las comunas de Talcahuano, Tomé, Penco y Hualpén y el Golfo de Arauco cuyo borde costero corresponde a las comunas de Coronel y Lota, existiendo incluso una isla poblada en dicho golfo, la isla Santa María perteneciente a la comuna de Coronel. En general es una zona de humedales, gran parte de los cuales ha sido rellenada a efecto de habilitar terrenos para viviendas.

Asociado a la geografía aparece una actividad logísticoportuario en el AMC muy relevante a nivel nacional con una presencia de 14 puertos marítimos, entre específicos y generales, mediante los cuales se reciben insumos y se despacha carga de exportación, principalmente forestal y pesquera. En esta área se ha consolidado una red logística regional con vinculación nacional, fundamentada en la actividad portuaria, con uno de los principales sistemas de puertos del sur pacífico, que se apoya en el sistema vial, portuario, ferroviario y aéreo existente de acuerdo al Plan Regional de Ordenamiento Territorial (PROT) Región del Biobío 2015-2030.

Esta actividad genera un importante y continuo tránsito de camiones de carga, el cual afecta la movilidad de las personas del AMC, con congestión, contaminación y accidentes. Ella también genera tránsito de carga ferroviaria proveniente de provincias vecinas y que atraviesa las comunas del área metropolitana. Asimismo, la geografía es fuente de riesgo por desastres naturales tales como tsunamis, desbordes de ríos y esteros, remoción de masa, lo que también ha condicionado la movilidad de las personas.

En términos sociodemográficos los niveles de pobreza y desempleo en algunas comunas son altos, con cifras que están sobre el promedio nacional.

Desde el punto de vista de actividades, hay comunas que claramente se perfilan como "comunas-dormitorio", tales como: Chiguayante, San Pedro de la Paz, Tomé, Lota, Hualqui o Hualpén. En otras, como Concepción, Talcahuano, Penco y Coronel existe una fuerte actividad de servicios, logístico portuario y sector pesquero.

En términos específicos, el uso del territorio o actividades promovidas por el PROT de la Región del Biobío 2015-2030 para el Área Metropolitana (MA-14) es el siguiente:

- Servicios urbanos y actividades culturales de escala metropolitana.

- Industria de gran envergadura.

- Logística, Portuaria y Pesquero (artesanal e industrial).

- Educación Superior e Investigación.

- Encadenamientos productivos asociados a nuevas tecnologías, innovación y creatividad.

- Turismo de negocios, Histórico Cultural, Turismo Aventura, Turismo de naturaleza, Turismo de sol y playa.

Todas estas actividades y complejidad condicionan las demandas y ofertas de movilidad por parte de los ciudadanos del AMC, las que no sólo se originan en función de las necesidades vinculadas al trabajo e ingresos familiares, estudio o trámites, sino también, de las oportunidades culturales, de esparcimiento y turismo, entre otras. En este contexto, es que se presenta la oportunidad de elaborar el primer Plan de Movilidad Sostenible incorporando la participación ciudadana con foco en la gestión pública eficaz.

\section{Metodología}

El Plan de Movilidad Sostenible fue requerido en 2017 por el Gobierno Regional del Biobío, teniendo como objetivo elaborar un plan para el Área Metropolitana de 
Concepción (AMC), con el fin de abordar las principales problemáticas y líneas de intervención en materia de movilidad e infraestructura de transporte del AMC. En función de ello, la Universidad del Bío-Bio llevó a cabo un trabajo dividido en cuatro etapas: fase preparatoria; diagnóstico; generación de propuestas; y propuesta del modelo de gestión y difusión.

A efecto de diseñar un plan de movilidad sostenible, de forma que incorpore el punto de vista social, es fundamental contar con las percepciones, opiniones, sensaciones e incluso emociones de los ciudadanos para obtener un diagnóstico socialmente validado. Para esto, la metodología empleada combinó la realización de talleres participativos y una encuesta a los participantes en cada una de las comunas que conforman el área metropolitana Por lo tanto, se produjeron datos cualitativos y cuantitativos que se complementan analíticamente. Los 11 talleres, contaron con la participación de diversos actores de la sociedad civil y ciudadanía en general. La convocatoria incluyó juntas de vecinos, agrupaciones de adultos mayores, agrupaciones deportivas, estudiantes secundarios, organizaciones culturales, sindicatos/gremios, y agrupaciones de discapacidad. El grupo total de personas que participaron en los talleres fue de 380 .

Si bien no se trató de una muestra estadística, se trabajó con una muestra cualitativa estructural (Hernández, Fernández y Baptista, 2008), basada en informantes claves. Por ello se buscó que esta fuera lo más representativa de la composición ciudadana de cada comuna y de los temas que se buscaba relevar, para ello, se determinaron ciertos criterios (variables) a cumplir: sexo; grupo etario; lugar de residencia (urbano-rural); nivel de ingresos; y situación de discapacidad.

Los talleres participativos, consideraron una metodología basada en principios del grupo de discusión (Figura 2), ya que se buscó construir colectivamente un relato ciudadano, entendido también como una forma de discurso social, mediante el cual aportar al diseño y mejoramiento del espacio urbano desde la mirada de sus protagonistas (Canales, 2006).

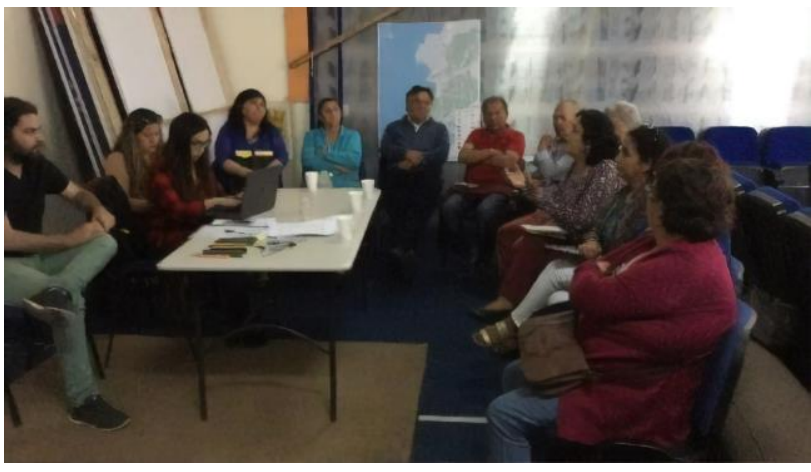

Figura 2. Taller Participativo.

Fuente: elaboración propia.

Adicionalmente, y a modo de realizar triangulación de instrumentos y contenidos, se aplicó un cuestionario a los 380 participantes en cada taller, el que buscó recabar información cuantificable respecto de la mirada individual y subjetiva de cada participante frente a los desafíos de la movilidad en el AMC. El instrumento recopiló, además, información de tipo sociodemográfica (edad, sexo, lugar de residencia, actividad, nivel de ingreso, composición hogar) y también referida a los viajes, modos, necesidades y percepciones surgidas durante el desplazamiento de las personas. Ahora, se debe recalcar que lo importante no era lo relativo a los orígenes y destinos, hay instrumentos que testean esta información, sino detectar las experiencias y sensaciones de las personas durante sus procesos de movilidad, algo relativamente inédito a nivel nacional.

El taller base se dividió en cuatro momentos: en el primero, los actores realizaron una descripción de sus rutas habituales y no habituales de desplazamiento (en el área metropolitana) junto con responder el cuestionario recién descrito; en el segundo momento se identificaron las principales problemáticas vinculadas al desplazamiento; en el tercero se profundizó en las percepciones y sensaciones generadas durante el desplazamiento, entendiendo que los componentes emocionales constituyen un indicador relevante a considerar en la planificación de hábitats eficientes; y finalmente, en el cuarto momento, se llevaron a cabo propuestas ciudadanas para sustentar una política pública fundamentada en los sentires colectivos y con arraigo territorial. 


\section{Principales Resultados}

A continuación, se presenta los resultados y hallazgos identificados en los talleres enfocados en el segundo y tercer momento de estos. Las distintas opiniones e ideas que surgieron de las discusiones grupales, apoyados por el software Atlas.ti, pasaron por un proceso de clasificación y agrupación en nubes de palabras lo que dio lugar a la consolidación de categorías de problemas. Las dimensiones de análisis de la información sistematizada fueron agrupadas en categorías emergentes de problemas, las que están directamente vinculadas a distintos modos de desplazamiento. Debido a lo anterior se proponen ocho categorías analíticas que agrupan los distintos problemas y dificultades que cada tipo de usuario enfrenta el desplazarse por el espacio metropolitano:

- Problema- Diseño y Planificación

- Problema- Transporte Público (Locomoción Colectiva)

- Problema- Transporte Público (Biotren)

- Problema- Ciclista

- Problema- Peatón

- Problema- Vehículo Particular

- Problema- Vehículo de Carga

- Problema- Aspectos Socioculturales

Problemas de diseño y planificación. Los conceptos más reiterativos fueron la mala conectividad interna en las comunas, argumentándose la existencia de un sistema de transporte que gira solamente en torno de la Capital Regional y Provincial (Concepción), no generándose así, alternativas para la conectividad al interior de las demás comunas. Esto genera la existencia de sectores sin locomoción pública, potenciados por un crecimiento inmobiliario desvinculado de los temas de conectividad, y un deficiente acceso a servicios, concentrados además en zonas puntuales del área metropolitana. A continuación se citan opiniones y comentarios de los ciudadanos, en relación con esta problemática identificada:
Falta transporte público en las poblaciones nuevas, o las que no son tan cercanas. Eso produce que cada familia tenga un vehículo y haga uso permanente de ello. (Taller Coronel)

Mala planificación urbana y de los servicios, están construyendo poblaciones nuevas sin servicios cerca por lo que tendrán que viajar al centro de la comuna, tampoco hay locomoción que los acerque. (Taller Penco)

Problemas de Transporte público. El transporte público puede ser dividido en dos categorías: locomoción colectiva que corresponde a buses urbanos y Biotren ${ }^{1}$.

Locomoción colectiva, Buses Urbanos. Dentro de esta categoría fue posible identificar problemas referidos al mal servicio brindado por los operadores. Allí se tocó aspectos como limpieza, frecuencia, costos del pasaje, imprudencias, poca preparación por parte de los conductores del transporte, falta de educación vial y situaciones de maltrato de los conductores del transporte hacia los usuarios, especialmente en el caso de adultos mayores, personas con discapacidad y estudiantes.

Mal servicio de transporte, principalmente a causa del maltrato y las maniobras peligrosas, rápidas de los conductores. Tanto los estudiantes como los adultos mayores manifiestan el mal trato de los conductores y discapacitados. (Taller Concepción)

Del mismo modo, la falta de educación se propone como un problema recurrente, producto del maltrato y la ocurrencia de situaciones desagradables al interior de los buses de la locomoción.

Locomoción colectiva, Biotren. En esta categoría destacaron los problemas de conectividad interna en las comunas producto de la división generada por la línea férrea, como es el caso de Coronel, San Pedro y Chiguayante. Esto supone una barrera para el adecuado tránsito y circulación al interior de las comunas, generando situaciones de congestión, cruces peligrosos, demoras, discriminación y marginación socioespacial.
${ }^{1}$ El Biotrén es un sistema de ferrocarril suburbano, el cual cubre gran
parte del área metropolitana de Concepción. Su recorrido abarca a las comunas de Concepción, Talcahuano, Hualpén, San Pedro de la Paz, Coronel, Chiguayante y Hualqui. 
La llegada del Biotren ha generado grandes problemas de movilidad interna, sectores excluidos producto de la línea férrea. (Taller Coronel)

La línea férrea divide a la ciudad en dos, hay mala conectividad al interior de la comuna. (Taller Chiguayante)

Problemas de ciclistas. Esta categoría agrupa una serie de problemas vinculados al uso de la bicicleta como medio de transporte. Uno relevante, es la ausencia de una red de ciclovías conectadas, de modo que quienes optan por desplazarse en bicicleta se ven enfrentados a una serie de condiciones adversas relacionados a ello. Así, se observa una importante demanda por mejorar el actual estado de la infraestructura dispuesta para el desplazamiento de ciclistas. Se menciona también como relevante, la falta de educación vial reflejadas en malas prácticas por parte de conductores, peatones y ciclistas.

Es peligroso movilizarse en bicicleta por la comuna, hay un solo lugar en la comuna donde hay ciclovías, los choferes no respetan al ciclista, pasan a muy alta velocidad uno anda con miedo. (Taller Hualqui)

También se menciona la ausencia de modos mixtos de transporte que den pie a la combinación de tipos de desplazamientos, cuestión que desincentiva el uso de la bicicleta a través del área Metropolitana.

Problemas de peatones. En lo referido al desplazamiento peatonal, el aspecto más destacado fue el de la inseguridad, cuestión que tiene directa relación con la mala evaluación del estado y uso de las veredas. Estas se encontrarían en una situación de tensión al soportar diversos tipos de desplazamiento, actividades económicas, infracciones vehiculares, perros callejeros, etc. Ello hace del caminar una actividad hostil que se acrecienta frente a una sociedad agresiva y falta de buena convivencia. El acceso al desplazamiento se hace inequitativo, afectando aún más a quienes se encuentran en situación de discapacidad o son adultos mayores.

Los adultos mayores no tienen la misma posibilidad de salir de sus hogares, producto del mal estado de las calles, y por la poca capacidad con que cuenta el transporte público para quienes tienen dificultades de desplazamiento. Desde que se sube al microbús. (Taller Concepción)
Problemas vehículo particular. Para el uso de vehículos particulares destacaron temas como la congestión, la falta de educación vial y la agresividad social. Al respecto, es fundamental precisar el reconocimiento de dos factores claves: uno por supuesto es la deficiente infraestructura frente al explosivo aumento de vehículos particulares, lo que conlleva a altos niveles de congestión. No obstante, también emerge como un problema relevante el comportamiento de los conductores, quienes con su imprudencia y desconsideración contribuirían a empeorar esta situación.

Ha habido un aumento explosivo de la población de la comuna, la falta de planificación urbana ha contribuido a generar excesiva congestión vehicular. (Taller Chiguayante)

Nuestra sociedad individualista, agresiva, poco empática. (Taller Hualpén)

Problemas vehículos de carga. Como era de esperar, los vehículos de carga también se identificaron como un problema, el cual se agudiza en algunas de las comunas más lejanas, tales como Hualqui y Santa Juana. Al respecto, se argumenta que las escasas rutas de acceso (como la ruta 160 , ruta 150 , ruta de la madera, entre otras) hacen de la presencia y circulación de transporte pesado un factor relevante en la congestión, la accidentabilidad, el excesivo tiempo de espera, el mal estado de los caminos rurales $y$, en definitiva, la generación de condiciones para hacer de las vías antes mencionadas peligrosas e inseguras. En las comunas más céntricas, en tanto, los problemas apuntan a la poca fiscalización en el cumplimiento de los horarios establecimiento para la carga y descarga de vehículo pesados, lo que genera situaciones de congestión y demoras.

Exceso de velocidad de camiones que transitan por la ruta de la madera. (Taller Santa Juana)

El transporte de los vehículos de carga pasa sin una restricción, no se respeta la reglamentación que controla la circulación de los vehículos de carga. (Taller Tomé)

Nuevamente la constatación de una sociedad agresiva y la falta de educación vial emergen como elementos negativos, requiriéndose fortalecer habilidades blandas en los usuarios del transporte. Ello con el objetivo de 
mejorar las relaciones de convivencia y hacer del desplazamiento a través de la metrópolis una experiencia positiva.

Problemas de índole sociocultural. Por último, los aspectos socioculturales se situaron como una categoría transversal que agrupa problemas ligados a hábitos y prácticas de los habitantes del área metropolitana. Entre ellos destacan el maltrato en que incurren los conductores del transporte público, además de la falta de educación para un buen trato, en el modo de relacionarse las personas entre sí. Esto apuntaría a las interacciones que se dan durante los desplazamientos, independiente del modo por el cual se opte, como origen de la mayor parte de los problemas que afectan la movilidad en el área metropolitana.

Hay una mala educación que es transversal, la condición de los choferes es un reflejo de lo que pasa en nuestra sociedad. (Taller Concepción)

Lo anterior, sumado a actitudes negativas de convivencia, tales como la imprudencia en la conducción, el abandono de perros en la calle, la acumulación de desechos y basura en espacios no habilitados revela la necesidad de educar no solo en temas de viabilidad, sino también de convivencia y corresponsabilidad para el desarrollo de la ciudad.
Por otro lado, respecto al cuestionario individual, se destaca entre algunos de los hallazgos de este instrumento los destinos de desplazamiento, siendo un $73,6 \%$ de ellos dentro de la comuna de referencia y un $26,4 \%$ a otros destinos del AMC. Por otra parte, la percepción de los principales problemas que enfrentan las personas al momento de desplazarse por el área metropolitana apuntó a las veredas en mal estado con un $62,3 \%$, la falta de mantención en las calles con un $51,2 \%$ y la mala calidad en el trato de los conductores con un $37,3 \%$ seguida de la inseguridad producida por la delincuencia y el vandalismo con un $30,2 \%$.

No obstante, y a propósito de cuestiones como la inseguridad, uno de los hallazgos y aportes más interesantes proporcionados por la encuesta es el referido a las sensaciones manifestadas por la ciudadanía durante su desplazamiento por el AMC. Para este fin se procedió a consultar de forma abierta respecto de la experiencia personal y subjetiva vivenciada por las personas al transportarse. Luego de ello, y con el fin de poder sistematizar este gran cúmulo de información, los datos fueron sometidos a un proceso de codificación que, mediante un análisis de contenido, redujo la variedad de respuestas a un número específico de categorías catalogadas como experiencias positivas o negativas. Los resultados se pueden ver en la Tabla 1:

\section{Tabla 1}

Experiencias de desplazamiento AMC

\begin{tabular}{lcc}
\hline & Experiencia & Porcentaje \% \\
\hline \multirow{2}{*}{ Experiencias Positivas } & Agrado & 12,7 \\
& Tranquilidad & 11,6 \\
& Relajo & 5,6 \\
\cline { 2 - 3 } Experiencias Negativas & Seguridad & 2,1 \\
\hline & Estrés & 16,2 \\
& Inseguridad/ Temor/ Desprotección & 33,1 \\
& Desagrado / Molestia / Mal Humor & 12,7 \\
& Rabia / Impotencia & 0,4 \\
& Preocupación & 1,4 \\
& Incomodidad & 1,1 \\
& Frustración / Pena & 3,2
\end{tabular}

Fuente: Universidad del Bío Bío (2018). Informe Propuesta Plan de Movilidad Sostenible Área Metropolitana Concepción. 
La Tabla 1 muestra cómo las experiencias de desplazamiento negativas (7) se presentan con mayor frecuencia (casi un 70\%) en las opiniones de los encuestados, figurando

la
inseguridad/temor/desprotección ampliamente como la experiencia más reiterativa con un $33,1 \%$, seguida del estrés con un $16,2 \%$. Recién en tercer lugar figura una experiencia positiva como es el agrado, con un $12,7 \%$ de las respuestas. No obstante, comparte puesto con el desagrado/molestia/mal humor.

Los datos de la encuesta son consistentes con los contenidos elaborados a partir de lo discutido en los talleres. En ellos, la identificación de los problemas, tenían asociado elementos emocionales como temor o estrés, lo que permite la encuesta es dimensionar el volumen individual de esas opiniones. Con ello, estamos afirmando, que tanto la dimensión colectiva del problema, como la individual son plenamente coincidentes respecto al efecto emocional que producen los problemas de la actual forma de movilidad de la ciudadanía.

Estos datos son reveladores ya que recogen la subjetividad asociada a la experiencia de moverse por la metrópolis, tratándose de variables poco (o en nada) consideradas en los planes o políticas públicas y que, sin embargo, podrían ser decisivas a la hora de entender cómo se habita la urbe, más allá de las mejoras en infraestructura y recursos. Es decir, ¿será sustentable construir, por ejemplo, más parques si la gente siente inseguridad o temor de circular por ellos? ¿Bastará solo con instalar más vías si no nos preocupamos del estrés experimentado por quienes circulan en ellas? ¿Cuáles son las consecuencias sicosomáticas respecto de ello? ¿Será valorado por las personas un transporte ambientalmente amigable que no satisfaga sus necesidades emocionales? ¿Qué oportunidades de mejorar estas experiencias cargadas de sensaciones negativas nuevas formas de transporte motorizados o no motorizados? Estas son algunas de las interrogantes que será interesante investigar a partir de las nuevas formas de mirar la ciudad que nos propone la movilidad sostenible.

\section{Conclusiones}

El Área Metropolitana de Concepción presenta un creciente desarrollo urbano que genera en forma natural problemas de movilidad no sostenible, por ejemplo: crecimiento sostenido del parque automotriz. Es decir, para comprender la complejidad creciente y sus problemas, es necesario realizar el análisis desde una perspectiva de movilidad urbana sostenible, enfocado en la interacción entre las dimensiones económica, ambiental y social involucradas. Esto requiere una adaptación a las complejidades de un sistema multifactorial de relaciones sociales y actividades productivas, en las que el desarrollo económico ha generado nuevas demandas de movilidad por parte de los ciudadanos, que han sido difíciles de satisfacer por la actual oferta de equipamiento, infraestructura y modos de transporte.

Este trabajo ha tenido como objetivo generar una reflexión enfocada en la dimensión social, a través del despliegue de un componente participativo como base para un plan de movilidad, específicamente en el análisis de un diagnóstico que responde a estos fines. Tal ejercicio, que puede parecer rutinario a la luz de otras políticas participativas, por ejemplo, en ámbitos sectoriales o de gobierno local, es inédito en lo referido a un plan de desarrollo de instrumentos territoriales supralocales o subregionales, lo cual le confiere un valor agregado necesario de destacar.

La participación, canalizada por la vía de talleres con enfoque de deliberación pública (Cohen, 2007; Elster, 2001) y la consulta a través de cuestionarios, ha significado explorar componentes diagnósticos estructurales y de la propia experiencia de los ciudadanos de este territorio. Con ello vemos la referencia a la importancia de los factores subjetivos emocionales vinculados (Tudela, Daziano y Carrasco, 2013), pero además la necesidad de que estos puedan ser considerados como insumos para incidir en la calidad de las políticas y de los servicios. Los participantes, además del diagnóstico, logran identificar propuestas que se relacionan a sus necesidades y experiencias, y que el plan a través del estudio descrito puede recoger e integrar, por ejemplo: el factor educativo con foco en el buen trato y la convivencia vial responsable. Uno de los aspectos que se pueden observar, es el juicio negativo a la movilidad actual en general, destacando los aspectos de tipo emocionalafectivos, lo que a su vez indica el fuerte compromiso personal y la cercanía que tiene este fenómeno en la experiencia cotidiana de las personas. Dicho de otro modo, no podría circunscribirse la movilidad solo al 
estudio de los flujos de transporte motorizados, puesto que integralmente, las sensaciones, emociones y afectos de los participantes están relacionados con las diversas formas que ella adquiere. Urge por tanto considerar estos aspectos a la hora de generar una planificación pertinente.

Al respecto, los ciudadanos no son solo usuarios de un sistema de movilidad, sino los que generan el ambiente social de estos procesos, condicionándolos y al mismo tiempo siendo afectados directamente por ellos en su calidad de vida diaria. Por ello, a los ciudadanos, es necesario considerarlos más que entidades que son trasladadas o circulan autónomamente por el sistema.

Por lo tanto, no solo se requiere mejorar la infraestructura, el modelo de transporte público o los incentivos al uso de medios alternativos. Existen requerimientos de tipos sociocultural, como mejorar la educación para la movilidad, integrar los elementos de movilidad sostenible en la planificación urbana y sobre lo anterior la instalación de mecanismos de gobernanza que permitan integrar las demandas ciudadanas en la planificación de los territorios.

Finalmente, se responde la pregunta de la investigación en el sentido que, si es posible, mediante un proceso participativo recoger elementos subjetivos, individuales y colectivos, asociados a los procesos de movilidad ciudadana, a efecto de incorporarlos en la formulación del plan. Asimismo, la metodología utilizada fue correspondiente y releva la importancia de la participación ciudadana como un componente central en el proceso de gestión para la concreción de una movilidad sostenible. Si bien esta lógica es incipiente en estos planes en Chile, es necesaria no solo porque permite levantar información sobre necesidades y expectativas, sino principalmente porque en el proceso los ciudadanos se posesionan y empoderan de un enfoque territorial, el cual requiere no solo de la técnica, sino también de una mirada desde la diversidad de usos y satisfacción de necesidades en un entorno global de complejidad creciente.

\section{Referencias}

Aguilar, L. (2014). Las dimensiones y los niveles de la gobernanza. Cuadernos de gobierno y administración pública, 1(1), 11-36. http://dx.doi.org/10.5209/rev CGAP.2014.v1.n1.451 $\underline{56}$

Alcántara, E. (2010). Análisis de la movilidad urbana. Espacio, medioambiente y equidad. Bogotá: Dirección de Análisis y Programación Sectorial de la Vicepresidencia de Infraestructura de CAF. https://www.caf.com/media/3155/An\%C3\%A1lisis m ovilidad urbana.pdf

Alessandri, A. (2014). La ciudad como privación y la reapropiación de lo urbano como ejercicio de la ciudadanía. Scripta Nova: Revista electrónica de geografía y ciencias sociales, 13(493), 1-14. http://dx.doi.org/10.1344/sn2014.18.14979
Aliste, E. (2014). Pensar los territorios del desarrollo: sustentabilidad y acción pública en el nombre de una ciudad imaginaria. Concepción (Chile), 1950-2010. EURE, 40(120), 91-110. http://dx.doi.org/10.4067/S0250$\underline{71612014000200005}$

Baño, R. (1998). Participación Ciudadana: Elementos Conceptuales. En E. Correa y M. Noé (Eds.), Nociones de una ciudadanía que crece (pp. 15-37). Santiago: Flacso Chile. http://flacsochile.org/biblioteca/pub/publicos/1998/li bro/002297.pdf\#page $=15$

Bourdin, A. (1999). Les services à la mobilité: structuration de la vie quotidienne et déplacements. Sèvres: Colloque mobilités territoires.

Canales, M. (2006). Metodologías de Investigación. Introducción a los Oficios. Santiago: Editorial LOM. 
Cerda, J. y Marmolejo, C. (2010). De la accesibilidad a la funcionalidad del territorio: Una nueva dimensión para entender la estructura urbano-residencial de las áreas metropolitanas de Santiago (Chile) y Barcelona (España). Revista de Geografía Norte Grande, (46), 527.

http://dx.doi.org/10.4067/S0718$\underline{34022010000200001}$

Cohen, J. (2007). Deliberative Democracy. In S. Rosenberg (ed.) Deliberation, Participation and Democracy Can the People Govern? New York: Macmillan.

Cunha, J. (2000) La movilidad intrarregional en el contexto de los cambios migratorios en Brasil en el período 1970-1991: el caso de la Región Metropolitana de São Paulo. Notas de Población, (70), 149-185. Disponible en

https://repositorio.cepal.org/bitstream/handle/1136 2/12693/NP70-05 es.pdf?sequence $=1$ \&isAllowed $=y$

Cunill, N. (1991). Participación Ciudadana. Dilemas y Perspectivas para la Democratización de los Estados Latinoamericanos. Caracas: Centro Latinoamericano de Administración Para el Desarrollo.

Dangond, C., Jolly, J., Monteoliva, A. y Rojas, F. (2011). Algunas reflexiones sobre movilidad urbana en Colombia desde la perspectiva del desarrollo humano. Papel Político, 16(2), 485-514. Disponible en http://www.scielo.org.co/pdf/papel/v16n2/v16n2a07 .pdf

De la Maza, G. (2008). Mecanismos de Participación Ciudadana en el Diseño, Implementación y Evaluación de Políticas Públicas. Santiago: Universidad de Los Lagos. Disponible en https://es.scribd.com/document/271061310/De-LaMaza-Gonzalo-2008-Mecanismos-de-ParticipacionCiudadana-en-El-Diseno-Implementacion-y-

Evaluacion-de-Politicas-Publicas

De la Maza, G. (2011). Espacio público y participación ciudadana en la gestión pública en Chile: Límites y posibilidades. Polis, 10(30), 45-75. http://dx.doi.org/10.4067/S071865682011000300003 .

Elster, J. (2001). La democracia deliberativa. Barcelona: Editorial Gedisa.
Escudero, N. (2004). La mobilité dans la ville, une composante essentielle du développement urbain durable (Tesis doctoral). Universidad Católica de Lovaina. Bélgica.

Espluga, J., Cebollada, A, y Miralles-Guash, C. (2008). Percepciones de la movilidad y participación ciudadana en la región metropolitana de Barcelona. Ciudad y Territorio Estudios Territoriales, 40(157), 499$510 . \quad$ Disponible en http://anycerda.org/congres/documentacio/postmetr opolis/docs/C/C8.pdf

Feria, J. (2008). Un ensayo metodológico de definición de las áreas metropolitanas en España a partir de la variable residencia-trabajo. Investigaciones Geográficas, (46), 49-68. Disponible en http://www.redalyc.org/pdf/176/17618757003.pdf

Fernández, V. (2014). Promoviendo un diseño urbano participativo: experiencias desde la práctica y la docencia. AUS, (15), 22-27. http://dx.doi.org/10.4206/aus.2014.n15-05

Garrido-Vergara, L., Valderrama, L. y Ríos, J. (2016). Democracia deliberativa, instituciones y participación ciudadana en América Latina. Revista de Ciencia Política, 54(2), 225-275. http://dx.doi.org/10.5354/0716-1077.2017.44807

Gasparini, A. \& Guidicini, P. (1990). Innovazione tecnologica e nuovo ordine urbano. Milán: Angeli.

Giraldo-Zuluaga, G. (2015). Ciudadanía: aprendizaje de una forma de vida. Educ. Educ. 18(1), 76-92. http://dx.doi.org/10.5294/edu.2015.18.1.5

Gobierno de Chile (2016). Chile Informe Nacional Habitat III. Disponible en http://www.habitat3.cl/wpcontent/uploads/2016/04/CHILE-INFORMENACIONAL-HABITAT-III-1.pdf

Gutiérrez, A. (2012). ¿Qué es la movilidad? Elementos para (re) construir las definiciones básicas del campo del transporte. Bitácora Urbano Territorial, 21(2), 61$74 . \quad$ Disponible en http://www.redalyc.org/pdf/748/74826255011.pdf

Herce, M. (2009). Sobre la movilidad en la ciudad. Propuestas para recuperar un derecho ciudadano. Barcelona: Editorial Reverté. 
Hernández, R., Fernandez, C. y Baptista, P. (2008) Metodología de la investigación. México: DF. McGrawHill Interamericana Editores.

Herrmann, M. (2016). Instrumentos de planificación y diseño urbano para promover al peatón en las ciudades: un estudio comparado entre Chile y Alemania. Urbano, (34), 48-57.

Hidalgo, R. y Arenas, F. (2009). Del país urbano al país metropolitano. Transformaciones recientes en las ciudades chilenas. En: HIDALGO, R.; DE MATTOS, C. y ARENAS, F. (editores.). Chile: Del país urbano al país metropolitano. Santiago de Chile: Pontificia Universidad Católica de Chile, Serie GeoLibros, 9-29. Disponible en http://geografia.uc.cl/images/serie GEOlibros/del pa is urbano/arenas hidalgo Del pais urbano al pais metropolitano.pdf

Instituto Nacional de Estadísticas. (2018). Compendio Estadístico 2018. Publicación Anual. Disponibl en https://www.ine.cl/docs/defaultsource/publicaciones/2018/bookcompendio2018.pdf ?sfvrsn $=5$

Janoschka, M. (2011). Geografías urbanas en la era del neoliberalismo. Una conceptualización de la resistencia local a través de la participación y la ciudadanía urbana. Investigaciones Geográficas, (76), 118-132. Disponibl

en http://www.scielo.org.mx/scielo.php?script=sci artte $\underline{x t \& p i d=S 0188-46112011000300009}$

Jiménez, C. (2008). La Gobernanza, un concepto problemático en América Latina. Foro Latinoamericano de Gobernanza. Disponible en http://www.institutgouvernance.org/es/analyse/fiche-analyse-327.html

Kaufman, V. \& Widmer, E. (2006). Motility and family dynamics: current issues and research agendas. Zeitschrift für Familienforschung, (18), 111-129. Disponible en https://www.researchgate.net/publication/37446534 Motility and Family Dynamics Current Issues and Research Agendas
Kaufman, V., Bergman, M. y Joye, D. (2004). Motility: Mobility as Capital. International Journal of Urban and Regional Research, 28(4), 745-756. https://doi.org/10.1111/i.0309-1317.2004.00549.x

Instituto Nacional de Estadísticas de Chile (2018). Censo Nacional de Población y Vivienda 2017. Disponible en https://www.ine.cl/estadisticas/censos/censos-depoblacion-y-vivienda

Lange, C. (2011). Dimensiones culturales de la movilidad urbana. INVI, 26(71), 87-106. http://dx.doi.org/10.4067/S071883582011000100004

Lévy, J. (2000). Les Nouveaux Espaces de la Mobilité. En M. Bonnet y D. Desjeux (Eds.), Les Territoires de la Mobilité (pp. 155-170). Paris: Presses Universitaires de France.

Maturana, F., Rojas, A. y Salas, R. (2017). Dinámicas espaciales y transición hacia la articulación de espacios metropolitanos. El caso de Temuco y su hinterland, Chile. Cuadernos Geográficos de la Universidad de Granada, 57(1), 132-154. Disponible en http://revistaseug.ugr.es/index.php/cuadgeo/article/ view/5628

Mello, A. (2017). Un procedimiento basado en la accesibilidad al diseño de planes estratégicos de la movilidad urbana: El caso de Brasil. EURE, 43(128), 1-

$27 . \quad$ Disponible en http://www.eure.cl/index.php/eure/article/view/171 $\underline{5 / 958}$

Mendoza, E. (2013). La movilidad urbana como modus operandi que edifica las ciudades. Economía, sociedad y territorio, 13(42), 571-575. Disponible en http://www.scielo.org.mx/scielo.php?script=sci_artte xt\&pid=S1405-84212013000200011

Miralles-Guash, C., Cebollada, A. y Requena, R. (2010). Estrategias de participación ciudadana en la gestión de la movilidad y el transporte. La Universidad Autónoma de Barcelona como ejemplo. Scripta Nova, Revista Electrónica de Geografía y Ciencias Sociales Universidad de Barcelona, 14 (331), s.n. Disponible en http://revistes.ub.edu/index.php/ScriptaNova/article $/$ view/1690 
Muñiz, I., Rojas, C., Busuldu, C., García, A., Filipi, M. y Quintana, M. (2016). Forma urbana y huella ecológica en el área metropolitana de Concepción (Chile). EURE, 42(127), 209-230. http://dx.doi.org/10.4067/S0250$\underline{71612016000300009}$

ONU (2016). Conferencia de las Naciones Unidas sobre la Vivienda y el Desarrollo Urbano Sostenible (Hábitat III). Disponible en http://www.habitat3.cl/wpcontent/uploads/2016/10/Nueva-Agenda-UrbanaHabitat-III.pdf

ONU-Habitat (2015). Movilidad. Disponible en https://es.unhabitat.org/temas-urbanos/movilidad/

Osorio, J. y García, J. (2016). Nuevas fuentes y retos para el estudio de la movilidad urbana. Cuadernos Geográficos, 53(3), 247-267. Disponible en http://www.redalyc.org/articulo.oa?id=17154972012

Gobierno Regional del Biobío (2015). Plan de Ordenamiento Territorial de la Región del Biobío. Disponible en http://erd.gorebiobio.cl/prot/

Poduje, I. (2008). Participación ciudadana en proyectos de infraestructura y planes reguladores. Temas de Agenda Pública, Pontificia Universidad Católica de Chile, (22). Disponible en https://politicaspublicas.uc.cl/wpcontent/uploads/2015/02/participacion-ciudadanaen-proyectos-de-infraestructura-y-planesreguladores.pdf

Quintero, J. (2017). Del concepto de ingeniería de tránsito al de movilidad urbana sostenible. Ambiente $y$ Desarrollo, 21(40), 57-72. https://doi.org/10.11144/Javeriana.ayd21-40.citm

Ramírez, B. (2007). La Geografía Regional: Tradiciones y Perspectivas Contemporáneas. Investigaciones Geográficas, (64), 116-133. Disponible en http://www.scielo.org.mx/scielo.php?script=sci_artte $\underline{x t \& p i d=S 0188-46112007000300008}$

Rolnik, R. (2009) La democracia en el filo de la navaja: límites y posibilidades para la implementación de una agenda de reforma urbana en Brasil. Eure 35(104), 7$27 . \quad$ http://dx.doi.org/10.4067/S0250$\underline{71612009000100001}$

Sagaris, L. y London, P. (2017). Autopistas, ciudadanía y democratización: la costanera norte y el acceso sur,
Santiago de Chile (1997-2007). EURE, 43(128), $127-$

$152 . \quad$ http://dx.doi.org/10.4067/S0250-

$\underline{71612017000100006}$

Salinas, E. y Perez, L. (2011). Procesos urbanos recientes en el Área Metropolitana de Concepción: transformaciones morfológicas y tipologías de ocupación. Revista Geografía Norte Grande, (49), 7997. http://dx.doi.org/10.4067/S0718$\underline{34022011000200006}$

Sánchez, L. y Gutierrez, A. (2014). Potencialidades de la participación en la construcción de ciudad desde intervenciones urbanas en asentamientos precarios. América Latina Hoy, (68), 119-136. http://dx.doi.org/10.14201/alh201468119136

Sanz, A. (1997). Movilidad y accesibilidad: un escollo para la sostenibilidad urbana. En La construcción de la ciudad sostenible. Disponible en http://habitat.aq.upm.es/cs/p3/a013.html

Scheneider, C. y Welp, Y. (2015). Diseños Institucionales y (des)equilibrios de poder: las instituciones de participación ciudadana en disputa. Revista Mexicana de Ciencias Políticas y Sociales, 60(224), 15-43. https://doi.org/10.1016/S0185-1918(15)30002-7

Silva, H. (2017). ¿Qué sucede con la movilidad en Chile? Plataforma Urbana. Disponible en http://www.plataformaurbana.cl/archive/2017/03/01 Lopinion-que-sucede-con-la-movilidad-en-chile

Truffello, R. e Hidalgo, R. (2015). Policentrismo en el Área Metropolitana de Santiago de Chile: reestructuración comercial, movilidad y tipificación de subcentros. EURE, 41(122), 49-73. http://dx.doi.org/10.4067/S0250$\underline{71612015000100003}$

Tudela, A., Daziano, R. y Carrasco, J. (2013). El Papel de los Factores Contextuales, Socioeconómicos y Sicológicos en la Elección de Modo. Un Estudio de Caso en Concepción. Ingeniería de Transporte, 17(01), 29-35. Disponible en http://www.ingenieriadetransporte.org/index.php/so chitran/article/view/136

Unión Europea (2017). Movilidad Urbana Sostenible. Política europea, práctica y soluciones. Bruselas: Unión Europea. https://doi.org/10.2832/66350 
Universidad del Bío-Bío, (2018). Propuesta de Plan de Movilidad Sostenible para el Área Metropolitana de Concepción.

Vega, P. (2016). Una década de planes de movilidad urbana sostenible en España 2004-2014. Anales de Geografía, 36(2), 351-372. https://doi.org/10.5209/AGUC.53589

Vergara, L., Rozas, M. y Zunino, H. (2014). Los imaginarios urbanos y la arquitectura de Puerto Varas. Encrucijada entre lo local y global. AUS, (14), 19-22. https://doi.org/10.4206/aus.2013.n14-05

Welp, Y. (2017) La participación ciudadana como compromiso democrático. Revista Mexicana de Derecho Electoral, (10), 97-121. http://dx.doi.org/10.22201/iij.24487910e.2016.10.11 122
Wiener, A. (1997) La ciudadanía como estrategia política. La Ventana. Revista de estudios de género. (5) 1422. Disponible

en http://revistalaventana.cucsh.udg.mx/index.php/LV/artic le/viewFile/777/752

World Business Council for Sustainable Development, [WBCSD]. (2001). Movilidad 2001: perspectiva general. Disponible en https://www.wbcsd.org/contentwbc/download/2639 $\not 33261$ 